\title{
Hyphenated NMR Methods in Natural Products Research, Part 2: HPLC-SPE-NMR and Other New Trends in NMR Hyphenation
}

\begin{abstract}
This review describes the principles and performance of a novel and highly promising hyphenated technique, HPLC-SPE-NMR, which is based on post-column analyte trapping by solid-phase extraction. The analytes are subsequently eluted from the SPE cartridges using deuterated solvents. This indirect HPLC-NMR hyphenation offers numerous advantages compared to direct HPLC-NMR methods. Multiple trapping leads to a dramatic increase of analyte amounts available for NMR, enabling acquisition of high-quality 2D NMR data within a short time. Other
\end{abstract}

new developments, including combination of solenoidal coil capillary flow-probes with microflow HPLC, are also discussed. Fast extract dereplication using these techniques enables focusing of isolation efforts on truly novel and promising natural products, based on precise structural data obtained with crude extracts or fractions.

\section{Key words}

Nuclear magnetic resonance · liquid chromatography · LC-NMR . HPLC-SPE-NMR · hyphenated techniques · microcoils · cryoprobes

\section{Introduction}

In the first part of this review [1], the use of direct NMR hyphenation in natural products research has been discussed. The direct HPLC-NMR methods were defined as methods in which the NMR data acquisition is performed on the HPLC column eluate [1]. This implies either a direct connection between the NMR spectrometer and the HPLC apparatus, or use of storage capillaries in which the HPLC eluate is temporarily stored prior to acquisition of NMR data.

Many useful results related to natural products have been obtained using direct HPLC-NMR methods [1] and the technique has been reviewed at various stages of its development [2], [3], [4], [5], [6], [7], [8], [9], [10], [11], [12], [13], [14], [15], [16], [17], [18]. Pharmaceutical, biomedical and other applications of NMR hyphenation have also been extensively reviewed [19], [20], [21],
[22], [23], [24], [25], [26], [27], [28], [29], [30]. However, the direct combination of state-of-the-art separation technology with the most powerful and versatile tool for structure elucidation of organic molecules in solution suffers from various practical problems. In short, these practical limitations are due to somewhat contradictory requirements of HPLC and NMR with respect to solvents and time-scale of the experiment, and thus compromises are necessary in order to hyphenate these two techniques. For example, ${ }^{1} \mathrm{H}$-NMR spectra are preferably acquired in deuterated solvents, but use of deuterated solvents for HPLC is expensive and the selection is limited. Some useful HPLC eluents, such as buffers with high ionic strengths, are directly undesirable in direct HPLC-NMR hyphenation. Gradient elution of HPLC columns, required in most separations, implies constantly changing magnetic susceptibility and field homogeneity in the NMR flowprobe, not to mention variations of analyte chemical shifts with solvent composition. 
On the other hand, the limited sensitivity of NMR detection means that HPLC-NMR experiments are usually acquired in the stopped-flow mode [1]. This can result in difficulties caused by diffusion and can limit the possibility of obtaining 2D NMR data to one peak per chromatogram. The latter difficulty is circumvented by use of capillary loops for temporary storage of chromatographic peaks [1]; however, only a few studies relying on the loop storage technique have been reported so far [1]. To sum up, the outcome of a direct HPLC-NMR experiment is largely determined by chromatography, both in terms of the solvent used and because analyte from one injection only is available for NMR.

The recently introduced automated HPLC-SPE-NMR technique makes use of an entirely different approach to HPLC-NMR. Here, chromatographic peaks eluted from a reversed-phase HPLC column are passed one by one through small solid-phase extraction columns (SPE cartridges) in order to remove the analyte from the HPLC mobile phase. The SPE cartridges are subsequently dried with nitrogen gas, and the analytes desorbed with a deuterated solvent for NMR spectroscopy. The whole process is carried out automatically under software control (Fig. 1).

\section{Early Combinations of Post-Column SPE with HPLC-NMR}

Solid-phase extraction is a standard method of sample preparation and analyte concentration prior to HPLC analysis [31]. Such analyte pre-concentration by SPE can also be combined on-line with HPLC-NMR analysis; for example, de Koning et al. used an SPE-HPLC-NMR experiment for the analysis of hop bitter acids [32].

The HPLC-SPE-NMR technique, where SPE is used after the HPLC separation, has its roots in the early phase-system switching (PSS) technique developed to solve mobile-phase and flow-rate incompatibilities in HPLC-MS. Kokkonen and colleagues were apparently the first to use post-column addition of water to the HPLC eluate in order to diminish the eluting power of the mobile phase and withhold the analyte on a trapping column prior to desorption to the MS interface [33]. Silica-based $C_{8}$ material was used in this HPLC-SPE-MS experiment both in the analytical and in the trapping columns [33]. With respect to NMR applications, Wilcox and Phelan reported already in 1986 a procedure consisting of a reversed-phase HPLC separation, collection of HPLC fractions using an automatic fraction collector, dilution of the frac- tions with water, solid-phase extraction of the diluted fractions using $\mathrm{C}_{18} \mathrm{SPE}$ cartridges, and elution of the analyte from the cartridges for ${ }^{1} \mathrm{H}-\mathrm{NMR}$ at $250 \mathrm{MHz}$ [34]. This off-line sequence of HPLC, SPE and NMR operations can be regarded as a prototype of modern HPLC-SPE-NMR experiments.

Griffiths and Horton [35] applied the concept of analyte concentration on a trapping column after water dilution of analytical column eluate [33] in connection with on-line NMR analysis. The analytes were desorbed from the trapping column with a back-flush of $\mathrm{CD}_{3} \mathrm{CN}$, but without prior drying of the trapping column [35]. Griffiths and Horton demonstrated that column trapping of chromatographic peaks leads to increased solute concentrations resulting in increased signal-to-noise ratios in the NMR spectra, showed the advantage of the use of deuterated solvents for analyte back-flush, and pointed out the similarity of their concept to the already introduced loop storage system [35]. The system of Griffiths and Horton can be regarded as an immediate forerunner of the state-of-the-art HPLC-SPE-NMR installations (Fig.1). Later, Nyberg et al. used SPE-NMR hyphenation, transferring analytes from SPE cartridges with a system of switching valves to the NMR flow-probe with $\mathrm{CD}_{3} \mathrm{OD}$, whereas the separation of sample constituents (Capsicum chinense fruit extract or Quillaja saponaria saponin fraction) was performed off-line [36], [37].

\section{Advantages of HPLC-SPE-NMR Hyphenation}

The signal-to-noise ratio in NMR spectra increases linearly with analyte amount but only with the square root of acquisition time. This emphasizes the importance of maximizing analyte amounts in the sensitive region of the NMR flow-cell. The sensitivity of detection in direct HPLC-NMR experiments will also depend on the ratio between the active volume of the NMR flow-cell and the peak elution volume (flow rate $\times$ peak width). Thus, in the majority of stopped-flow HPLC-NMR experiments performed with conventional columns with internal diameter of $4.6 \mathrm{~mm}$ or larger [1], only a small fraction of the analyte eluted from the column contributes to the NMR signal. For example, it can be calculated that for a Gaussian chromatographic peak with a standard deviation of $0.2 \mathrm{~min}$ (and hence the base-width of $0.8 \mathrm{~min}$ ), eluted at a flow rate of $1 \mathrm{~mL} / \mathrm{min}$, the maximal percentage of the analyte present in the sensitive region of an NMR flow-probe is $23.6 \%$, $11.9 \%$ or $6.0 \%$ for flow-probes with sensitive volumes of 120,60

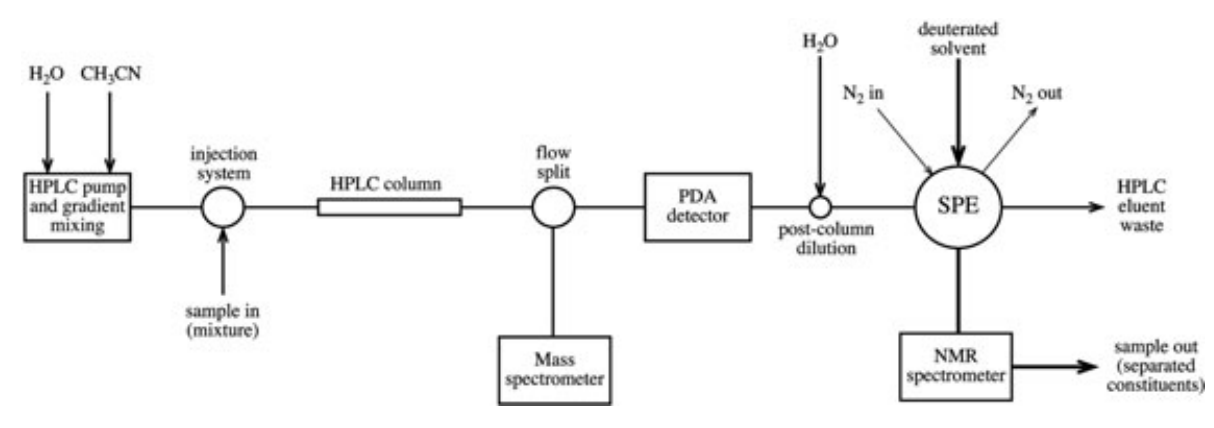

Fig. 1 Schematic description of an HPLCSPE-NMR system. After separation of the mixture components on a reversed-phase HPLC column with non-deuterated eluents, the chromatogram is recorded using a photodiode array detector, a mass spectrometer, or both. The HPLC column eluate is then diluted with water and the analytes present in the chromatographic peaks are trapped on individual solid-phase extraction (SPE) cartridges. The trapping is governed by signals from the PDA or the MS detector. The SPE cartridges are then dried with a stream of nitrogen gas, and the analytes eluted with a deuterated solvent into the NMR flow-probe. 
or $30 \mu \mathrm{L}$, respectively. If the peak-width is reduced four-fold (peak standard deviation $0.05 \mathrm{~min}$ and the base-width $0.2 \mathrm{~min}$ ), the percentage of the analyte present at the center of the peak in the above-mentioned sensitive volumes increases to $77.0 \%$, $45.2 \%$ and $23.6 \%$, respectively. This emphasizes the importance of sharp peaks in direct HPLC-NMR experiments.

On the other hand, if the analyte can be trapped on an SPE cartridge quantitatively and eluted with about $30 \mu \mathrm{L}$ of a solvent, most of the analyte will enter the NMR flow-cell and contribute to the NMR signal if a $30-\mu \mathrm{L}$ flow-cell is used. In such a case, there will be an additional advantage of intrinsically higher mass sensitivity of the small-volume flow-cell. The only diminishing factor left will be the ratio between the sensitive volume and the total volume of the NMR cell (equal to about $1 / 2$ ). This focusing of the analyte band is the basis of the HPLC-SPE-NMR technique. Additional benefits come from the use of pure deuterated solvents for cartridge elution, which will render solvent suppression easier or even unnecessary. Last but not least, multiple peak trapping may give a very substantial increase of the analyte amount available for NMR.

Standard HPLC-SPE-NMR installations (Fig. 1) use $2 \times 10 \mathrm{~mm}$ SPE trapping cartridges with a dead volume of about $30 \mu \mathrm{L}$ that focus analytes eluted from standard HPLC columns into an NMR probe with a sensitive volume of $30 \mu \mathrm{L}$. Polystyrene- and/or divinylbenzene-based resins or $\mathrm{C}_{18}$-bonded silica is normally used as the SPE material, and $\mathrm{CD}_{3} \mathrm{CN}, \mathrm{CD}_{3} \mathrm{OD}$ or $\mathrm{CDCl}_{3}$ as the eluting solvent. The prerequisites for successful operation of the HPLC-SPE-NMR system are 1) that the analyte is essentially quantitatively retained on the SPE cartridge, and 2) that it is quantitatively stripped from the cartridge as a sharp band, essentially with the solvent front. In order to increase affinity of compounds eluted from reversed-phase HPLC columns to the SPE material, dilution of the eluate with water is used prior to passage through the SPE cartridges, and the ratio of the post-column dilution is an adjustable parameter for optimization of HPLC-SPE-NMR experiments. Other post-column eluate modifications, such as $\mathrm{pH}$ adjustment, are in principle possible but have not yet been reported in conjunction with HPLC-SPE-NMR. Although a number of successful applications of the HPLC-SPE-NMR approach have been published (see below), quantitative studies of trapping and elution efficiency of various classes of natural products have yet to be reported.

\section{Applications of HPLC-SPE-NMR}

The first published study utilizing the automated HPLC-SPENMR technique described the identification of several flavonoids, rosmarinic acid and carvacrol in crude acetone extract of oregano using 1D ${ }^{1} \mathrm{H}-\mathrm{NMR}$ spectra as well as TOCSY, HMQC and HMBC experiments [38]. The HPLC separation was performed on a $150 \times 4.6 \mathrm{~mm} \mathrm{C}_{18}$ column, analytes were trapped on a divinylbenzene-type polymer, and the spectra recorded with a cryogenic probe-head [38]. Other published studies describe identification of radical-scavenging flavonoids from extracts of Rhaponticum carthamoides (Asteraceae) separated on a $100 \times 7.8 \mathrm{~mm} \mathrm{C}_{18}$ column and employing polystyrene resin SPE cartridges [39], and structure determination of coumaric esters of iridoid glycosides from a pur- ified fraction of Harpagophytum procumbens (Pedaliaceae), separated on a $250 \times 2 \mathrm{~mm} \mathrm{C}_{18}$ column and trapped on $\mathrm{C}_{18}$-silica or poly(divinylbenzene) [40]. The latter work demonstrated the complexity of trapping efficiency and analyte recovery as a function of SPE stationary phase and eluting solvent [40].

Subsequent reports described the identification of flavonoids and cardenolides from various parts of Kanahia laniflora (Apocynaceae) separated on a $150 \times 4.6 \mathrm{~mm} \mathrm{C}_{18}$ column and trapped on $\mathrm{C}_{18}$ SPE cartridges [41], and identification of 17 compounds, mainly isoflavonoids, present in a crude extract of Smirnowia iranica (Fabaceae) roots [42]. In the latter study, a linear accumulation of an isoflavan on $\mathrm{C}_{18}$ SPE material in seven repeated trappings was demonstrated [42], illustrating the advantages of the multiple trapping approach; similar multiple trapping with a coumarin present in the root extract of Croton membranaceus (Euphorbiaceae) showed that poly(divinylbenzene) SPE material is more effective than $C_{18}$ material under the conditions used [43] (Fig. 2). Moreover, inversion recovery experiments showed that the relaxation times determined in $\mathrm{CD}_{3} \mathrm{CN}$, a commonly used solvent for cartridge elution, are much longer than those in $\mathrm{CDCl}_{3}$ [43]. Thus, not only SPE trapping and elution parameters, but also NMR acquisition parameters should be carefully optimized on a case-to-case basis for optimal performance of hyphenated NMR experiments. Constituents of a phenolic fraction of olive oil [44], lignans from Phyllanthus urinaria (Euphorbiaceae) [45], and oxygenated diterpenes from Rosmarinus officinalis (Labiatae) [46] were also analyzed using HPLC-SPE-NMR.

At present, the HPLC-SPE-NMR technique has been demonstrated to work well with many natural products classes including flavonoids, flavonoid glycosides and other glycosides, iridoids, various groups of terpenoids, simple phenolics, lignans and other phenylpropanoids, cardenolides, and alkaloids [38], [39], [40], [41], [42], [43], [44], [45], [46], [47]. Moreover, applications related to water and soil analysis [48] and drug metabolism [49], [50] have been reported. Xu and Alexander used HPLC-SPE-NMR in conjunction with a $150 \times 10 \mathrm{~mm} \mathrm{C}_{18}$ column to concentrate trace constituents for NMR analysis; since SPE trapping necessitates post-column water addition to the column eluate, a high-capacity retention and mixing device was introduced in order to achieve a sufficient dilution ratio of the high eluent flow $(5 \mathrm{~mL} /$ min) emerging from the column used [51].

Thus, even though the number of studies utilizing HPLC-SPENMR is still rather limited compared to those employing direct HPLC-NMR methods [1], its advantages are clearly apparent. The combined benefits of analyte concentration and accumulation and use of deuterated solvents enable acquisition of high quality 2D NMR data from multiple peaks, including minor peaks present in very complex chromatograms, without compromising the chromatographic separation process. Access to less-sensitive 2D NMR experiments such as NOESY/ROESY and availability of ${ }^{13} \mathrm{C}$ chemical shifts (from HSQC and HMBC experiments) allow rigorous structure elucidation of rather complex natural products directly from extracts and mixtures (Figs. 3 and 4). The large analyte amounts available by multiple trapping allow the recording of NMR spectra with excellent signal-to-noise ratios without solvent peak suppression, increasing the robustness of the experiment and eliminating possible suppression artifacts 
(Fig. 5). The fact that chemical shift data are obtained in well-defined solvents (as opposed to mixed HPLC solvents) opens the possibility for the use of databases for computer-aided spectra interpretation.

For these reasons, HPLC-SPE-NMR is envisaged to be a prime future tool for extract dereplication. Cryogenically cooled probes result in a further sensitivity gain and were applied in several of the above-mentioned studies [38], [49]. The HPLC-SPE-NMR studies reported thus far have been performed using $400 \mathrm{MHz}$ [38], [39], [45], [46], $500 \mathrm{MHz}$ [49], [51] or $600 \mathrm{MHz}$ magnets [40], [41], [42], [43], [44], [48], [50], but an increase in routinely available field
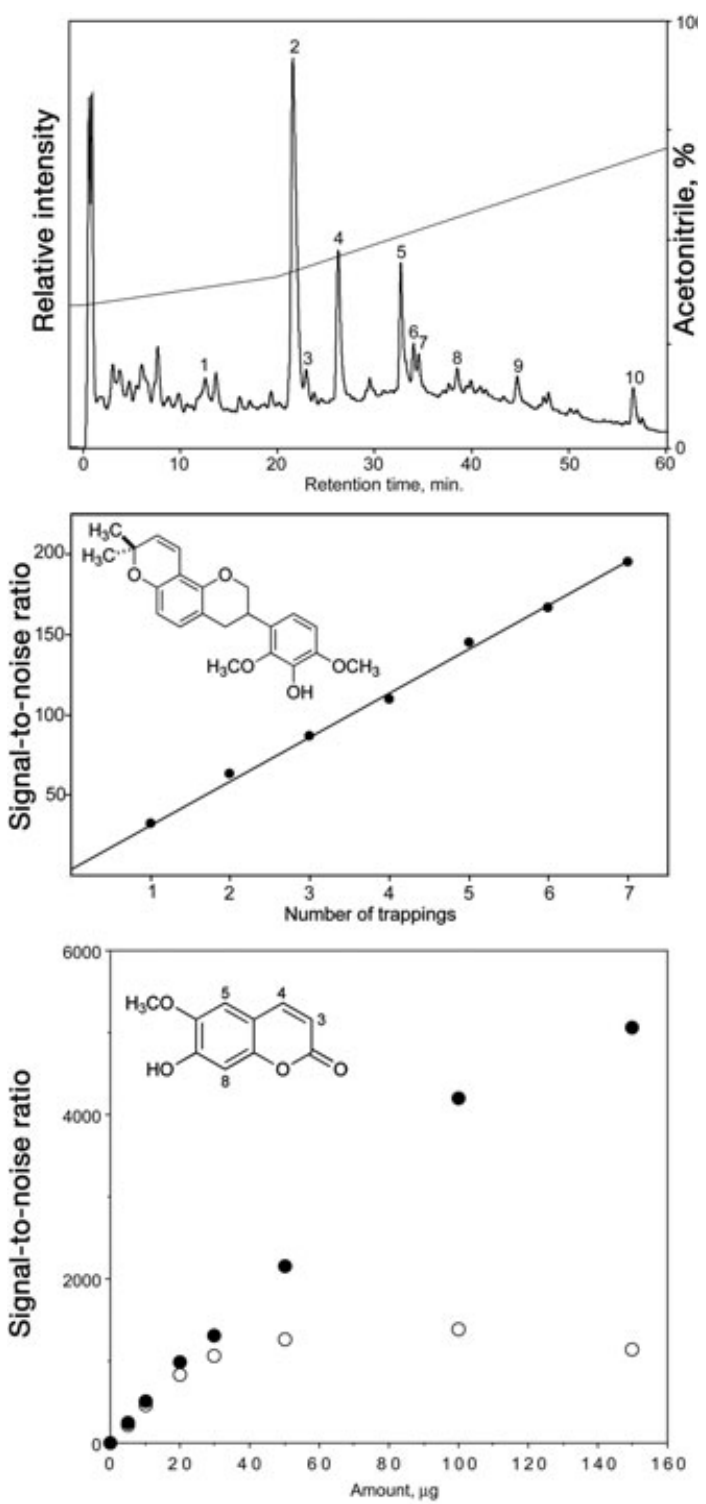

Fig. 2 Multiple trapping in HPLC-SPE-NMR experiments. Top: HPLC chromatogram of a crude extract of Smirnowia iranica roots (150 $\times$ $4.6 \mathrm{~mm} \mathrm{C}_{18}$ column eluted with $\mathrm{CH}_{3} \mathrm{CN}$ gradient in $\mathrm{H}_{2} \mathrm{O}$ ). Center: Linear improvement of ${ }^{1} \mathrm{H}-\mathrm{NMR}$ signal-to-noise ratio during seven trappings of the minor peak 10 from the above chromatogram on a $2 \times 10 \mathrm{~mm}$ $\mathrm{C}_{18}$ SPE cartridge. Bottom: Illustration of preferred trapping of a coumarin on poly(divinylbenzene) SPE cartridges (filled circles) as compared to $\mathrm{C}_{18}$ SPE cartridges (open circles). All spectra were recorded at 600 $\mathrm{MHz}$ and in $\mathrm{CD}_{3} \mathrm{CN}$, using a 30- $\mu \mathrm{L}$ flow-probe. Reproduced from [42] and [43] by permission of the American Chemical Society and John Wiley and Sons, respectively.
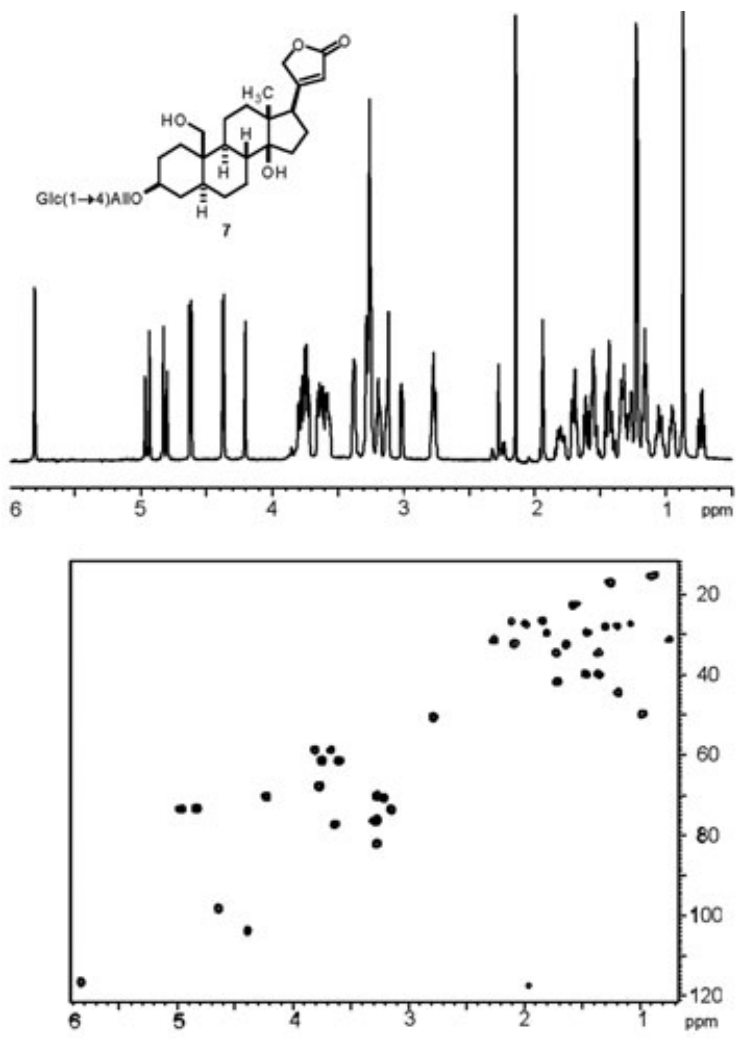

Fig. 3 1D ${ }^{1} \mathrm{H}-\mathrm{NMR}$ spectrum (top) and HSQC spectrum (bottom) of a $5 \alpha$-cardenolide present in Kanahia laniflora. The spectra were recorded in the HPLC-SPE-NMR mode following eight trappings on a $2 \times 10 \mathrm{~mm}$ $\mathrm{C}_{18}$ SPE cartridge $\left(150 \times 4.6 \mathrm{~mm} \mathrm{C}_{18}\right.$ column eluted with $\mathrm{CH}_{3} \mathrm{CN}$ gradient in $\mathrm{H}_{2} \mathrm{O}, 600 \mathrm{MHz}, 30-\mu \mathrm{L}$ cell). Reproduced from [41] by permission of the American Chemical Society.

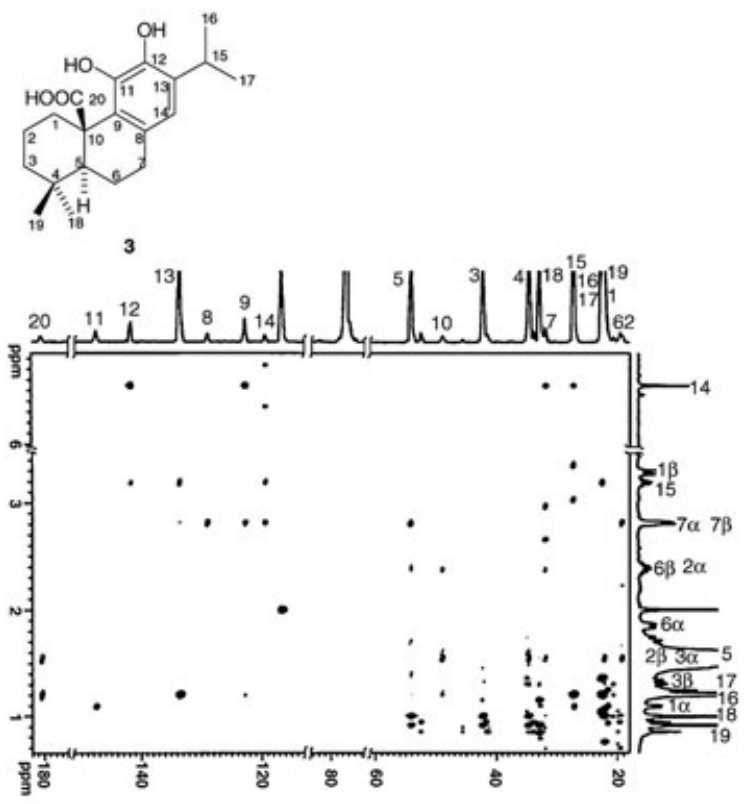

Fig. 4 HMBC spectrum recorded in the HPLC-SPE-NMR mode from an extract of Rosmarinus officinalis (Lamiaceae) following single trapping on a $2 \times 10 \mathrm{~mm}$ polystyrene-type SPE cartridge $\left(150 \times 4.6 \mathrm{~mm} \mathrm{C}_{18}\right.$ column eluted with $\mathrm{CH}_{3} \mathrm{CN}$ gradient in $\mathrm{H}_{2} \mathrm{O}, 400 \mathrm{MHz}, 120-\mu \mathrm{L}$ cell, cartridge eluted with $\mathrm{CDCl}_{3}$ ). Reproduced from [46] by permission of Elsevier. 

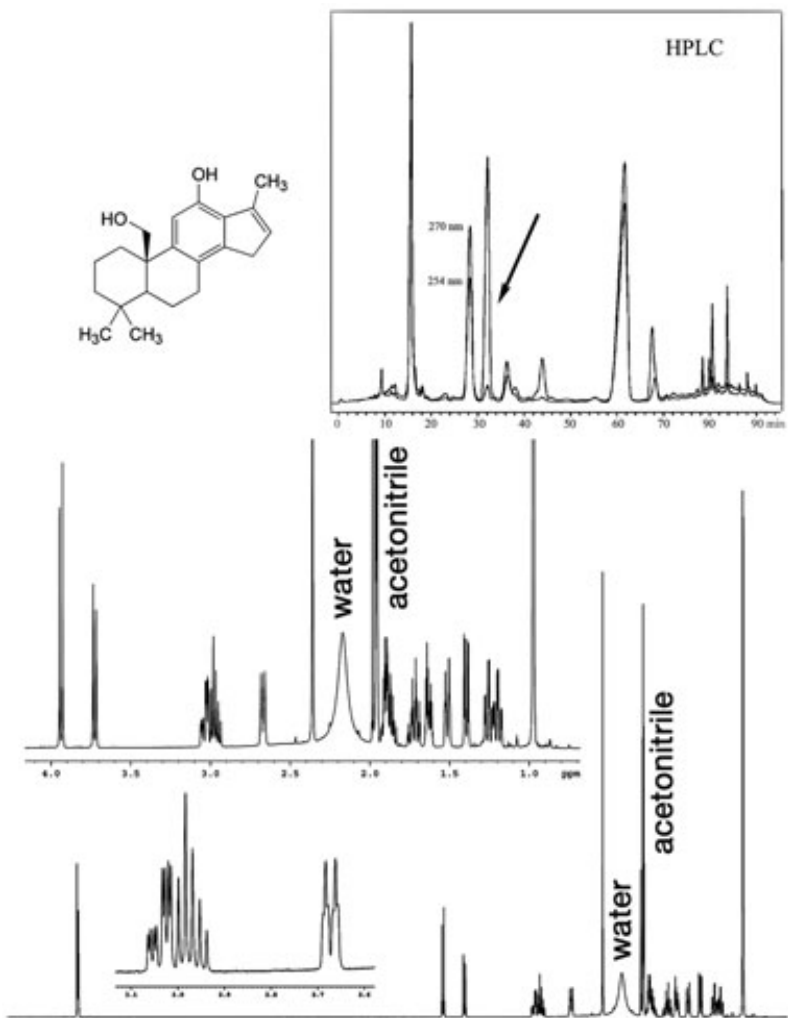

\begin{tabular}{lllllllllllllll}
\hline .0 & 7.5 & 7.0 & 6.5 & 6.0 & 5.5 & 5.0 & 4.5 & 4.0 & 3.5 & 3.0 & 2.5 & 2.0 & 1.5 & 1.0
\end{tabular}

Fig. $5600 \mathrm{MHz}{ }^{1} \mathrm{H}-\mathrm{NMR}$ spectrum obtained in the HPLC-SPE-NMR mode of a diterpene present in the ethyl acetate extract of roots of Perovskia atriplicifolia (Lamiaceae). The crude extract (1.5 mg per injection) was separated on a $150 \times 4.6 \mathrm{~mm} \mathrm{C}_{18}$ column with $\mathrm{CH}_{3} \mathrm{CN}$ gradient in $\mathrm{H}_{2} \mathrm{O}$. The arrow points to the peak analyzed (six trappings on a $2 \times$ $10 \mathrm{~mm} \mathrm{C}_{18}$ SPE cartridge, elution with $\mathrm{CD}_{3} \mathrm{CN}, 30-\mu \mathrm{L}$ cell NMR flow-cell, no solvent peak suppression). Unpublished work from the author's laboratory.

strengths is expected as the cost of high-field magnets drops. No systematic comparisons have yet been published, but $30-\mu \mathrm{L}$ (active volume) flow-probes together with $2 \times 10 \mathrm{~mm}$ SPE cartridges are believed to be preferable and were used in most of the aforementioned investigations [38], [40], [41], [42], [43], [44], [50].

Obviously, the success of HPLC-SPE-NMR experiment depends on trapping efficiency and very polar compounds are not expected to be trapped on reversed-phase materials. Also, very apolar compounds may be difficult to elute from the SPE cartridges with $\mathrm{CD}_{3} \mathrm{CN}$ [50] and the use of other solvents such as $\mathrm{CDCl}_{3}$ may be necessary. However, most classes of natural products appear to have suitable trapping and elution properties and even alkaloids eluted from HPLC columns with acid-containing eluents as charged species were trapped on polystyrene-type cartridges [47]. Moreover, an extension of the range of SPE materials used for HPLC-SPE-NMR experiments and further optimization of trapping parameters [52] as well as of flow-cell design can be envisaged.

\section{Hyphenation of Other Techniques with HPLC-SPE-NMR}

Standard HPLC-SPE-NMR installations include a photodiode array detector monitoring HPLC separation and triggering SPE trappings (Fig. 1), and hence the technique is sometimes referred to as HPLC-UV-SPE-NMR or HPLC-DAD-SPE-NMR. Thus, UV spectra are available along with the NMR data.

Already in the case of direct HPLC-NMR hyphenations [1], most of reported studies are supported by mass spectrometry, either using a separate HPLC-MS installation, or by introducing a split in the effluent flow and acquiring MS data simultaneously with the NMR data (HPLC-NMR-MS). It is of course possible to combine MS on-line with HPLC-SPE-NMR [38], [40], [49] (Fig. 1). This extended hyphenation is useful not only by making MS or MS/MS data available simultaneously with the NMR data, but also because trapping of non-UV-absorbing constituents can be triggered by signals provided by MS (total ion or single ion monitoring). Furthermore, it can be mentioned that evaporative light scattering detection (ELS), already used in connection with stopped-flow HPLC-NMR [53], could be used to trigger trapping in a putative HPLC-ELS-SPE-NMR experiment.

In principle, other spectroscopic techniques such as CD [16], [54], [55], [56], or for special applications IR [57], might be hyphenated with HPLC-SPE-NMR. Chirality determination of natural products is especially important and CD spectra recorded with SPEeluted material would benefit from increased concentration, use of pure solvents and the possibility of calibration of analyte concentration from the NMR response. Hyphenation of bioassays with HPLC-SPE-NMR is another exciting possibility [46].

\section{Other New Trends in NMR Hyphenation}

In an attempt to increase the sensitivity of NMR data acquisition, attention has been directed towards miniaturization of NMR coil design [58]. Small probe-volumes facilitate enclosing of a larger proportion of the total sample volume within the sensitive region of the probe (improved observe factor) and achieving maximal filling of the coil detection volume with the sample (improved filling factor). Moreover, the sensitivity per mass unit of miniaturized Helmholtz (saddle) coils used for vertical NMR tubes as well as vertical flow-cells used for direct HPLC-NMR hyphenation and for HPLC-SPE-NMR increases with reduction of coil length and diameter [59]. For these reasons, the $30-\mu \mathrm{L}$ (sensitive volume) flow-probes used for HPLC-SPE-NMR experiments have a higher mass sensitivity than the $120-\mu \mathrm{L}$ probes normally used for on-flow and stopped-flow HPLC-NMR.

Vertical capillary probes (with Helmholtz coils) having sensitive volumes down to $2.5 \mu \mathrm{L}$ have already been used for some time instead of normal NMR tubes [58], [60]. However, additional sensitivity gain can be achieved with probes consisting of a solenoid wrapped around a horizontal capillary [58], [59], [61], [62]. The sensitivity of such probes is inversely proportional to the coil diameter and very short radiofrequency pulses at low power are possible with these microcoils, which can be as small as $50 \mu \mathrm{m}$ in diameter.

The superior performance of solenoidal microcoils for the identification of natural products has been demonstrated using direct analyte injection [63], [64], [65], [66]. However, the main potential of microcoils is in their combination with microflow liquid 
chromatography, resulting in capillary HPLC-NMR [67], [68], [69], [70], [71], [72], [73], [74]. Stopped-flow capillary HPLCNMR was recently used for the identification of isoflavonoids in plant material [75]. Use of microcoil NMR detectors together with microbore or capillary HPLC separations, which operate at considerably higher analyte concentrations in elution bands, is a very promising new development within HPLC-NMR that enables high-throughput applications [76]. Further improvements are expected from use of micromachined coils [77] and multiple coil probes [69], [78], [79]. Miniaturization, SPE interfaces as well as the already mentioned cryogenic probe technology [80], [81], [82] are expected to continue to improve the sensitivity of HPLC-NMR experiments. Both the cryoprobes and the microcoil probes show a capability of making direct observation of ${ }^{13} \mathrm{C}$ NMR spectra (Fig. 6) feasible with analyte amounts that can be achieved in the HPLC-NMR mode, especially when combined with SPE. In addition, microcoils facilitate NMR detection in electro-driven capillary separations [83], [84], [85], [86], [87].

Since analyte quantification is an important aspect of NMR hyphenation, use of the ERETIC method (Electronic REference To access In vivo Concentrations), originally introduced for internal calibration of in vivo NMR spectra [88], is of interest. The method, which relies on the generation of a synthetic reference signal with defined frequency and amplitude, was subsequently adapted for solution studies [89], [90], [91], and can be used as a means of quantification of HPLC-NMR data.

Another new development is the use of ultra-fast (single scan) acquisition of 2D NMR data relying on spatial encoding of spin interactions [92], [93], [94]; although this approach suffers from serious sensitivity limitations, its applicability for macro-scale on-flow LC-NMR has been demonstrated [95].

\section{Conclusions}

The HPLC-SPE-NMR technique is a very powerful tool for on-line extract dereplication, enabling routine access to high-quality 2D NMR data without distortion of the HPLC separation of the remaining extract constituents. In contrast to direct HPLC-NMR ex-

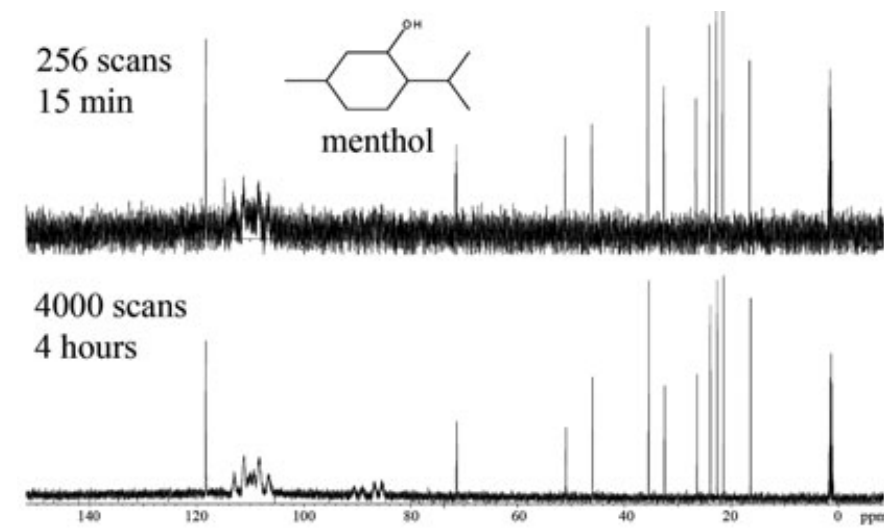

Fig. 6 Direct ${ }^{13} \mathrm{C}$ observation using solenoidal capillary probe (cell volume $5 \mu \mathrm{L}$, sensitive volume $1.5 \mu \mathrm{L}, 330 \mu \mathrm{g}$ of menthol, $150 \mathrm{MHz}$ observation frequency); broad signals between 80 - 120 ppm are probe background. Reproduced from [71] by permission of the American Chemical Society. periments, the amount of analyte available for NMR studies is not limited to that provided by a single injection to the HPLC column. The broad applicability of the technique to natural products has already been demonstrated, and the technique is envisaged to be an increasingly important analytical platform for natural products research. HPLC-SPE-NMR and other modern, hyphenated NMR techniques are expected to provide an increasing number of rigorously determined natural product structures directly from extracts and isolates, including compounds present in minor amounts. These developments will enable highthroughput extract analysis for the selection of those of extract constituents that deserve isolation and purification on a preparative scale, and in perspective on-line combinations of separation, automatic structure elucidation and bioassays.

\section{References}

1 Jaroszewski JW. Hyphenated NMR methods in natural products research part 1: direct hyphenation. Planta Med 2005; 71: 691 - 700

${ }^{2}$ Hostettmann K, Wolfender JL, Rodriguez S. Rapid detection and subsequent isolation of bioactive constituents of crude plant extracts. Planta Med 1997; 63: 2-10

${ }^{3}$ Wolfender JL, Ndjoko K, Hostettmann K. LC/NMR in natural products chemistry. Curr Org Chem 1998; 2: 575-96

${ }^{4}$ Wolfender JL, Rodriguez S, Hostettmann K. Liquid chromatography coupled to mass spectrometry and nuclear magnetic resonance spectroscopy for the screening of plant constituents. J Chromatogr A 1998; 794: $299-316$

${ }^{5}$ Hostettmann K, Terreaux C. Search for new lead compounds from higher plants. Chimia 2000; 54: $652-7$

${ }^{6}$ Wolfender JL, Terreaux C, Hostettmann K. The importance of LC-MS and LC-NMR in the discovery of new lead compounds from plants. Pharm Biol Suppl 2000; 38: 41 - 51

${ }^{7}$ Bobzin SC, Yang S, Kasten TP. Application of liquid chromatographynuclear magnetic resonance spectroscopy to the identification of natural products. J Chromatogr B 2000; 748: 259-67

${ }^{8}$ Bobzin SC, Yang S, Kasten TP. LC-NMR: a new tool to expedite the dereplication and identification of natural products. J Ind Microbiol Biotechnol 2000; 25: $342-5$

${ }^{9}$ Vogler B, Spring O. Application of LC-NMR measurements in phytochemical analysis. Recent Research Develop Phytochemistry 2000; 4: 207-22

${ }^{10}$ Hostettmann K, Wolfender JL, Terreaux C. Modern screening techniques for plant extracts. Pharm Biol Suppl 2001; 39: 18 - 32

${ }^{11}$ Wolfender JL, Ndjoko K, Hostettmann K. The potential of LC-NMR in phytochemical analysis. Phytochem Anal 2001; 12: 2-22

12 Sanvoss M. Application of LC-NMR and LC-NMR-MS hyphenation to natural products analysis. In: On-line LC-NMR and related techniques. Albert K, editor. Chichester: Wiley \& Sons; 2002. p. 111-28.

${ }^{13}$ Hostettmann K, Marston A. Twenty years of research into medicinal plans: results and perspectives. Phytochem Rev 2002; 1: 275 - 85

14 Wolfender JL, Ndjoko K, Hostettmann K. Liquid chromatography with ultraviolet absorbance-mass spectrometric detection and with nuclear magnetic resonance spectroscopy: a powerful combination for the on-line structural investigation of plant metabolites. J Chromatogr A 2003; 1000: $437-55$

${ }^{15}$ Wolfender JL, Ndjoko K, Hostettmann K. Application of LC-NMR in the structure elucidation of polyphenols. In: Methods in polyphenol analysis Santos-Buelga C, Williamson G, editors. Cambridge: The Royal Society of Chemistry; 2003. p. 128-56.

${ }^{16}$ Bringmann G, Lang G. Full absolute stereostructures of natural products directly from crude extracts: the HPLC-MS/MS-NMR-CD 'triad'. Prog Mol Subcell Biol 2003; 37: 89-116

17 Urban S, Separovic F. Developments in hyphenated spectroscopic methods in natural product profiling. Front Drug Des Discov 2005; 1: $113-66$

${ }^{18}$ Exarchou V, Krucker M, van Beek TA, Vervoort J, Gerothanassis IP, Albert K. LC-NMR coupling technology: recent advancements and applications in natural products analysis. Magn Reson Chem 2005; 43: $681-7$ 
${ }^{19}$ Albert K. On-line use of NMR detection in separation chemistry. J Chromatogr A 1995; 703: $123-47$

${ }^{20}$ Korhammer SA, Bernreuther A. Hyphenation of high-performance liquid chromatography (HPLC) and other chromatographic techniques (SFC, GPC, GC, CE) with nuclear magnetic resonance (NMR): a review. Fresenius J Anal Chem 1996; 354: 131 -5

${ }^{21}$ Lindon JC, Nicholson JK, Wilson ID. The development and application of coupled HPLC-NMR spectroscopy. Adv Chromatogr 1996; 36: 315-82

${ }^{22}$ Albert K. Liquid chromatography-nuclear magnetic resonance spectroscopy. J Chromatogr A 1999; 856: 199-211

${ }^{23}$ Albert K, Dachtler M, Glaser T, Händel H, Lacker T, Schlotterbeck G et al. On-line coupling of separation techniques to NMR. J High Resolut Chromatogr 1999; 22: 135-43

${ }^{24}$ Lindon JC, Nicholson JK, Wilson ID. Directly coupled HPLC-NMR and HPLC-NMR-MS in pharmaceutical research and development. J Chromatogr B 2000; 748: 233-58

${ }^{25}$ Wilson ID. Multiple hyphenation of liquid chromatography with nuclear magnetic resonance spectroscopy, mass spectrometry and beyond. J Chromatogr B 2000; 892: 315-27

26 Albert K, editor. On-line LC-NMR and related techniques. Chichester: Wiley \& Sons; 2002.

27 Corcoran O, Spraul M. LC-NMR-MS in drug discovery. Drug Discov Today 2003; 8: 624-31

${ }^{28}$ Elipe MVS. Advantages and disadvantages of nuclear magnetic resonance spectroscopy as a hyphenated technique. Anal Chim Acta 2003; 497: 1 - 25

${ }^{29}$ Cardoza LA, Almeida VK, Carr A, Larive CK, Graham DW. Separation coupled with NMR detection. Trends Anal Chem 2003; 22: 766 - 75

${ }^{30}$ Wann MH. Application of LC-NMR in pharmaceutical analysis. In: Handbook of pharmaceutical analysis by HPLC. Ahuja S, Dong MV, editors. Amsterdam: Elsevier; 2005. Vol. 6: p. 569-79.

${ }^{31}$ Pyrzynska K, Pobozy E. On-line coupling of solid phase extraction sample processing with high-performance liquid chromatography. Crit Rev Anal Chem 2002; 32: 227-43

${ }^{32}$ De Koning JA, Hogenboom AC, Lacker T, Strohschein S, Albert K, Brinkman UAT. On-line trace enrichment in hyphenated liquid chromatography-nuclear magnetic resonance spectroscopy. J Chromatogr A 1998; 813: 55-61

${ }^{33}$ Kokkonen PS, Niessen WMA, Tjaden UR, van der Greef J. Phase-system switching in liquid chromatography/continuous-flow fast-atom-bombardment mass spectrometry. Rapid Commun Mass Spectrom 1991; 5: $19-24$

${ }^{34}$ Wilcox CD, Phelan RM. The use of solid-phase extraction columns to effect simple offline LC/MS, LC/NMR, and LC/FTIR. J Chromatogr Sci 1986; $24: 130-3$

${ }^{35}$ Griffiths L, Horton R. Optimization of LC-NMR. III. Increased signal-tonoise ratio through column trapping. Magn Reson Chem 1998; 36: $104-9$

${ }^{36}$ Nyberg NT, Baumann H, Kenne L. Application of solid-phase extraction coupled to an NMR flow-probe in the analysis of HPLC fractions. Magn Reson Chem 2001; 39: 236 - 40

${ }^{37}$ Nyberg NT, Baumann H, Kenne L. Solid-phase extraction NMR studies of chromatographic fractions of saponins from Quillaja saponaria. Anal Chem 2003; 75: 268 - 74

${ }^{38}$ Exarchou V, Godejohann M, van Beek TA, Gerothanassis IP, Vervoort J. LC-UV-solid-phase extraction-NMR-MS combined with a cryogenic flow probe and its application to the identification of compounds present in Greek oregano. Anal Chem 2003; 75: 6288-94

39 Miliauskas G, van Beek TA, de Waard P, Venskutonis RP, Sudhölter EJR. Identification of radical scavenging compounds in Rhaponticum carthamoides by means of LC-DAD-SPE-NMR. J Nat Prod 2005; 68: $168-72$

${ }^{40}$ Seger C, Godejohann M, Tseng LH, Spraul M, Girtler A, Sturm S et al. LC-DAD-MS/SPE-NMR hyphenation, a tool for the analysis of pharmaceutically used plant extracts: identification of isobaric glycoside regioisomers from Harpagophytum procumbens. Anal Chem 2005; 77 : 878-85

${ }^{41}$ Clarkson C, Stærk D, Hansen SH, Jaroszewski JW. Hyphenation of solid-phase extraction and nuclear magnetic resonance: application of HPLC-DAD-SPE-NMR to identification of constituents of Kanahia laniflora. Anal Chem 2005; 77: 3547 -53

42 Lambert M, Stærk D, Hansen SH, Sairafianpour M, Jaroszewski JW. Rapid extract dereplication using HPLC-SPE-NMR: Analysis of isoflavonoids from Smirnowia iranica. J Nat Prod, in press
${ }^{43}$ Lambert M, Stærk D, Hansen SH, Jaroszewski JW. HPLC-SPE-NMR hyphenation in natural products research: optimization of analysis of Croton membranaceus extract. Magn Reson Chem 2005; 43: 771 - 7

${ }^{44}$ Christophoridou S, Dais P, Tseng LH, Spraul M. Separation and identification of phenolic compounds in olive oil by coupling high-performance liquid chromatography with postcolumn solid-phase extraction to nuclear magnetic resonance spectroscopy (LC-SPE-NMR). J Agric Food Chem 2005; 53: 4667-79

${ }^{45}$ Wang CY, Lee SS. Analysis and identification of lignans in Phyllanthus urinaria by HPLC-SPE-NMR. Phytochem Anal 2005; 16: 120-6

${ }^{46}$ Pukalskas A, van Beek TA, de Waard P. Development of a triple hyphenated HPLC-radical scavenging detection-DAD-SPE-NMR system for the rapid identification of antioxidants in complex plant extracts. J Chromatogr A 2005; 1074: $81-8$

47 Jaroszewski JW. HPLC-SPE-NMR: A productivity tool for determination of natural products in plant material. In: Magnetic resonance in food science, the multivariate challenge. Engelsen SB, Belton PS, Jacobsen HJ, editors. Cambridge: The Royal Society of Chemistry; 2005. p. $39-46$.

48 Simpson AJ, Tseng LH, Simpson MJ, Spaul M, Braumann U, Kingery WL et al. The application of LC-NMR and LC-SPE-NMR to compositional studies of natural organic matter. Analyst 2004; 129: 1216-22

${ }^{49}$ Godejohann M, Tseng LH, Braumann U, Fuchser J, Spraul M. Characterization of a paracetamol metabolite using on-line LC-SPE-NMR-MS and a cryogenic NMR probe. J Chromatogr A 2004; 1058: 191 -6

${ }^{50}$ Sandvoss M, Bardsley B, Beck TL, Lee-Smith E, North SE, Moore PJ et al. HPLC-SPE-NMR in pharmaceutical development: capabilities and applications. Magn Reson Chem 2005; 43: 762 - 70

$51 \mathrm{Xu}$ F, Alexander AJ. The design of an on-line semi-preparative LC-SPENMR system for trace analysis. Magn Reson Chem 2005; 43: 776-82

52 Poole CF, Gunatilleka AD, Sethuraman R. Contributions of theory to method development in solid-phase extraction. J Chromatogr A 2000; 885: 17-39

53 Petritis K, Gillaizeau I, Elfakir C, Dreux M, Petit A, Bongibault N et al. Evaporative light scattering detection for on-line monitoring of stopped-flow liquid chromatography-nuclear magnetic resonance analysis of compounds with weak or no chromophore groups. J Sep Sci 2002; 25: $593-600$

${ }^{54}$ Bringmann G, Messer K, Wohlfarth M, Kraus J, Dumbuya K, Rückert M. HPLC-CD on-line coupling in combination with HPLC-NMR and HPLCMS/MS for the determination of the full absolute stereostructure of new metabolites in plant extracts. Anal Chem 1999; 71: 2678-86

${ }^{55}$ Mistry N, Roberts AD, Tranter GE, Francis P, Barylski I, Ismail IM et al. Directly coupled chiral HPLC-NMR and HPLC-CD spectroscopy as complementary methods for structural and enantiomeric isomer identification: application to atracurium besylate. Anal Chem 1999; 71 : $2838-43$

${ }^{56}$ Bringmann G, Messer K, Saeb W, Peters EM, Peters K. The absolute configuration of (+)-isoshinanolone and in situ LC-CD analysis of its stereoisomers from crude extracts. Phytochemistry 2001; 56: 387 - 91

${ }^{57}$ Somsen GW, Gooijer C, Brinkman UAT. Liquid chromatography-Fourier transform infrared spectrometry. J Chromatogr A 1999; 856: 213 42

${ }^{58}$ Lacey ME, Subramanian R, Olson DL, Webb AG, Sweedler JV. High-resolution spectroscopy of sample volumes from $1 \mathrm{~nL}$ to $10 \mu \mathrm{L}$. Chem Rev 1999; 99: $3133-52$

${ }^{59}$ Hoult DI, Richards RE. Signal-to noise ratio of nuclear magnetic resonance experiment. J Magn Reson 1976; 24: 71 -85

${ }^{60}$ Schlotterbeck G, Ross A, Hochstrasser R, Senn H, Kühn T, Marek D et al. High-resolution capillary tube NMR. A miniaturized $5-\mu \mathrm{L}$ high-sensitivity TXI probe for mass-limited samples, off-line LC NMR, and HT NMR. Anal Chem 2002; 74: 4464 - 71

61 Peck TL, Magin RL, Lauterbur PC. Design and analysis of microcoils for NMR spectroscopy. J Magn Reson B 1995; 108: 114-24

${ }^{62}$ Olson DL, Peck TL, Webb AG, Magin RL, Sweedler JV. High-resolution microcoil ${ }^{1} \mathrm{H}$ NMR for mass-limited, nanoliter-volume samples. Science 1995; 270: 1967-70

${ }^{63} \mathrm{Hu}$ JF, Yoo HD, Williams CT, Garo E, Cremin PA, Zeng L et al. Miniaturization of the structure elucidation of novel natural products - two trace antibacterial acylated caprylic alcohol glycosides from Arctostaphylos pumila. Planta Med 2005; 71: 176-80

${ }^{64} \mathrm{Hu}$ JF, Garo E, Yoo HD, Cremin PA, Zeng L, Goering MG et al. Application of capillary-scale NMR for the structure determination of phytochemicals. Phytochem Anal 2005; 16: 127-33 
${ }^{65}$ Yoo HD, Cremin PA, Zeng L, Garo E, Williams CT, Lee CM et al. Suaveolindole, a new mass-limited antibacterial indolosesquiterpene from Greenwayodendron suaveolens obtained via high-throughput natural products chemistry methods. J Nat Prod 2005; 68: 22 -4

${ }^{66}$ Wolfender JL, Queiroz EF, Hostettmann K. Phytochemistry in the microgram domain - an LC-NMR perspective. Magn Reson Chem 2005; 43: $697-709$

${ }^{67}$ Wu N, Webb A, Peck TL, Sweedler JV. On-line NMR detection of amino acids and peptides in microbore LC. Anal Chem 1995; 67: 3101 - 7

${ }^{68}$ Subramanian R, Kelley WP, Floyd PD, Tan ZJ, Webb AG, Sweedler JV. A microcoil NMR probe for coupling microscale HPLC with on-line NMR spectroscopy. Anal Chem 1999; 71: 5335-9

69 Wolters AM, Jayawickrama DA, Sweedler JV. Microscale NMR. Curr Opin Chem Biol 2002; 6: 711-6

70 Jayawickrama DA, Sweedler JV. Hyphenation of capillary separations with nuclear magnetic resonance spectroscopy. J Chromatogr A 2003; 1000: 819-40

${ }^{71}$ Olson DL, Norcross JA, O'Neil-Johnson M, Molitor PF, Detlefsen DJ, Wilson AG et al. Microflow NMR: concepts and capabilities. Anal Chem 2004; 76: 2966 - 74

72 Sandvoss M, Roberts AD, Ismail IM, North SE. Direct on-line hyphenation of capillary liquid chromatography to nuclear magnetic resonance spectroscopy: practical aspects and application to drug metabolite identification. J Chromatogr A 2004; 1028: 259-66

${ }^{73}$ Hentschel P, Krucker M, Grynbaum MD, Putzbach K, Bischoff R, Albert K. Determination of regulatory phosphorylation sites in nanogram amounts of a synthetic fragment of ZAP-70 using microprobe NMR and on-line coupled capillary HPLC-NMR. Magn Reson Chem 2005; 43: $747-54$

${ }^{74}$ Lewis RJ, Bernstein MA, Duncan SJ, Sleigh CJ. A comparison of capillary-scale LC-NMR with alternative techniques: spectroscopic and practical considerations. Magn Reson Chem 2005; 43: 783 -9

75 Xiao HB, Krucker M, Putzbach K, Albert K. Capillary liquid chromatography-microcoil ${ }^{1} \mathrm{H}$ nuclear magnetic resonance spectroscopy and liquid chromatography-ion trap mass spectrometry for on-line structure elucidation of isoflavones in Radix astragali. J Chromatogr A 2005; 1067: 135-43

${ }^{76}$ Bailey NJC, Marshall IR. Development of ultrahigh-throughput NMR spectroscopic analysis utilizing capillary flow NMR technology. Anal Chem 2005; 77: 3947 - 53

77 Walton JH, de Ropp JS, Shutov MV, Goloshevsky AG, McCarthy MJ, Smith RL et al. A micromachined double-tuned NMR microprobe. Anal Chem 2003; 75: 5030-6

${ }^{78}$ Macnaughtan MA, Hou T, Xu J, Raftery D. High-throughput nuclear magnetic resonance analysis using a multiple coil flow probe. Anal Chem 2003; 75: 5116-23

${ }^{79}$ Wang H, Ciobanu L, Edison AS, Webb AG. An eight-coil high-frequency probehead design for high-throughput nuclear magnetic resonance spectroscopy. J Magn Reson 2004; 170: $206-12$
${ }^{80}$ Styles P, Soffe NF, Scott CA, Cragg DA, Row F, White DJ et al. A high-resolution NMR probe in which the coil and preamplifier are cooled with liquid helium. J Magn Reson 1984; 60: 397 - 404

81 Spraul M, Freund AS, Nast RE, Withers RS, Maas WE, Corcoran O. Advancing NMR sensitivity for LC-NMR-MS using a cryoflow probe: application to the analysis of acetaminophen metabolites in urine. Anal Chem 2003; 75: 1546-51

82 Kovacs H, Moskau D, Spraul M. Cryogenically cooled probes - a leap in NMR technology. Prog Nucl Magn Reson Spectrosc 2005; 46: 131 - 55

${ }^{83}$ Kautz RA, Lacey ME, Wolters AM, Foret F, Webb AG, Karger BL et al. Sample concentration and separation for nanoliter-volume NMR spectroscopy using capillary isotachophoresis. J Am Chem Soc 2001; 123 : $3159-60$

${ }^{84}$ Wolters AM, Jayawickrama DA, Larive CK, Sweedler JV. Capillary isotachophoresis/NMR: extension to trace impurity analysis and improved instrument coupling. Anal Chem 2002; 74: 2306-13

85 Jayawickrama DA, Sweedler JV. Dual microcoil NMR probe coupled to cyclic CE for continuous separation and analyte isolation. Anal Chem 2004; 76: 4894-900

${ }^{86}$ Wolters AM, Jayawickrama DA, Sweedler JV. Comparative analysis of a neurotoxin from Calliostoma canaliculatum by on-line capillary isotachophoresis $/{ }^{1} \mathrm{H}$ NMR and diffusion ${ }^{1} \mathrm{H}$ NMR. J Nat Prod 2005; 68: $162-7$

87 Webb AG. Nuclear magnetic resonance coupled microseparations. Magn Reson Chem 2005; 43: 688-96

88 Barantin L, Le Pape A, Akoka S. A new method for absolute quantification of MRS metabolites. Magn Reson Med 1997; 38: 179-82

${ }^{89}$ Akoka S, Barantin L, Trierweiler M. Concentration measurement by proton NMR using the ERETIC method. Anal Chem 1999; 71: 2554-7

${ }^{90}$ Akoka S, Trierweiler M. Improvement of the ERETIC method by digital synthesis of the signal and addition of a broadband antenna inside the NMR probe. Instr Sci Technol 2002; 30: 21 -9

91 Silvestre V, Goupry S, Trierweiler M, Robins R, Akoka S. Determination of substrate and product concentrations in lactic acid bacterial fermentations by proton NMR using the ERETIC method. Anal Chem 2001; 73: $1862-8$

92 Frydman L, Lupulescu A, Scherf T. Principles and features of singlescan two dimensional NMR spectroscopy. J Am Chem Soc 2003; 125: $9204-17$

${ }^{93}$ Pelupessy P. Adiabatic single scan two-dimensional NMR spectroscopy. J Am Chem Soc 2003; 125: 12345-50

${ }^{94}$ Shrot Y, Shapira B, Frydman L. Ultrafast 2D NMR spectroscopy using a continuous spatial encoding of the spin interactions. J Magn Reson 2004; 171: $163-70$

${ }^{95}$ Shapira B, Karton A, Aronzon D, Frydman L. Real-time 2D NMR identification of analytes undergoing continuous chromatographic separation. J Am Chem Soc 2004; 126: 1262 - 5 